\title{
Expression of carbonic anhydrase III and skeletal muscle remodeling following selective denervation
}

\author{
HE HUANG $^{1 *}$, YANLING ZHAO ${ }^{2 *}$, XILIANG SHANG $^{3}$, XUEYUAN LIU $^{1}$ and HUIMIN REN ${ }^{4}$ \\ ${ }^{1}$ Department of Neurology, Tenth People's Hospital of Tongji University, Shanghai 200072; ${ }^{2}$ Department of \\ Geriatrics, Shanghai First People's Hospital Affiliated to Shanghai Jiaotong University, Shanghai 200080; \\ ${ }^{3}$ Department of Sports Medicine; ${ }^{4}$ Institute of Neurology, Fudan University, Shanghai 200040, P.R. China
}

Received December 21, 2016; Accepted July 31, 2017

DOI: $10.3892 / \mathrm{mmr} .2017 .7644$

\begin{abstract}
Carbonic anhydrase III (CAIII) is expressed selectively in type I (slow-twitch) myofibers. To investigate the association between changes in the expression of CAIII and skeletal muscle structure following denervation, the present study stained adjacent sections of skeletal muscle for ATPase and immunohistochemically for CAIII. In addition, differences in the protein expression and phosphatase activity of CAIII were examined by western blot and phosphatase staining between rat soleus and extensol digitorum longus (EDL) muscles, which are composed of predominantly slowand fast-twitch fibers, respectively. Upon denervation, the EDL muscle showed more pronounced structural changes, compared with the soleus muscle. There was a transformation from fast to slow fibers, and a concomitant increase in fibers positive for CAIII. Following denervation, the protein expression of CAIII initially increased and then decreased in the soleus muscle, whereas the protein expression of CAIII in the EDL muscle increased gradually with time. In contrast to the protein changes, phosphatase activity in the soleus and EDL muscles decreased significantly following denervation. These results indicated that, following denervation, changes in the expression of CAIII were associated with myofiber remodeling. Specifically, the change in the expression of CAIII reflected the conversion to type I myofibers, suggesting the importance of CAIII in resistance to fatigue in skeletal muscle.
\end{abstract}

Correspondence to: Mr. He Huang, Department of Neurology, Tenth People's Hospital of Tongji University, 301 Middle Yanchang Road, Shanghai 200072, P.R. China

E-mail: morton162@hotmail.com

Professor Huimin Ren, Institute of Neurology, Fudan University, 12 Middle Wulumuqi Road, Shanghai 200040, P.R. China

E-mail: renhm188@163.com

${ }^{*}$ Contributed equally

Key words: carbonic anhydrase III, selective denervation, skeletal muscle remodeling, protein expression, phosphatase activity

\section{Introduction}

Carbonic anhydrase (EC4.2.1.1; CAIII) is considered a biological marker or therapeutic target for skeletal muscle disorders in a variety of pathophysiological conditions. Changes in the expression of CAIII, or the modification of CAIII by carbonylation or oxidation occur in Duchenne's muscular dystrophy, chronic widespread pain and skeletal muscle disuse (1-3). In previous years, various epidemiological, pathological and molecular investigations have demonstrated that CAIII is also involved in the development of myasthenia gravis (MG) (4), a neuromuscular autoimmune disorder caused by anti-acetylcholine receptor antibodies. Our previous investigations (4) showed that the protein and gene expression levels of CAIII were significantly decreased in the skeletal muscles of patients with MG. As the severity of MG increased, the decrease in the expression of CAIII also increased. As CAIII is expressed mainly in slow-twitch fibers (type I myofibers), exhibiting oxidative metabolism and having a low contraction speed and high resistance to fatigue, it was hypothesized that the changes in CAIII reflect abnormalities of type I myofibers in patients with MG. This may be the molecular mechanism underlying muscle fatigability. Compared with other carbonic anhydrase family isozymes, CAIII shows unique characteristics in activity and tissue distribution (5). Firstly, CAIII is a multifunctional enzyme with unique phosphatase activity in addition to lower specific hydrase activity ( $\sim 1 \%$ of CAII). Secondly, CAIII is expressed at high levels in tissues with in high metabolic activity, including fat, liver tissues and skeletal muscles. CAIII constitutes $\sim 10 \%$ of the soluble protein in the cytoplasm of slow-twitch fibers, but is largely absent from fast-twitch fibers. In rodents, the levels of CAIII in skeletal muscles change with age according to the development of innervation and contractile activity (6). Specifically, the level of CAIII increases sharply with age, reaching the peak adult level at puberty. The levels of CAIII are then maintained for a time, but eventually decline with aging (7). Comparative analysis has revealed a correlation between changes in the levels of CAIII and the development of skeletal muscle contractility (6).

CAIII may be involved in resistance to fatigue and its levels are affected by the innervation state $(8,9)$. For example, neuromuscular dysfunction caused by anti-acetylcholine 
receptor autoantibodies in $\mathrm{MG}$, denervation, or cross innervation, have an effect on the expression of CAIII. In previous studies, the expression of CAIII following denervation was examined by analyzing its overall protein and mRNA levels in skeletal muscles. However, whether changes in the levels of CAIII resulted from changes in expression in the original type I myofibers or from remodeling of skeletal muscle were not clarified. Additionally, denervation animal models are constructed predominantly by transecting the sciatic nerve. This surgery leads to rapid and complete denervation, but also causes dysfunction of almost the entire hind limb and impairs the blood supply to the musculature. These effects interfere with the examination of denervation-induced changes to skeletal muscle (10).

In the present study, to investigate the association between changes in the expression of CAIII and skeletal muscle characteristics, a highly selective denervation technique was used, which reduced the interference caused by changes in limb activity and blood supply. This technique involves transection of the nerves innervating the soleus (slow-twitch) and extensor digitorum longus (EDL; fast-twitch) muscles. In this model, specific skeletal muscles were denervated, but the movement and blood supply of the affected limb were maintained. This enables the first investigation, to the best of our knowledge, of the association between skeletal muscle remodeling and the expression of CAIII in serial sections.

\section{Materials and methods}

Animals and reagents. Male adult Sprague-Dawley rats, weighing 160-180 g, were obtained from the Experimental Animal Center of Shanghai Medical College, Fudan University (Shanghai, China). The rats had free access to food and water, and were subjected to standard conditions of humidity $(50 \pm 10 \%)$, temperature $\left(25 \pm 2^{\circ} \mathrm{C}\right)$ and a 12 -h light/dark cycle. All experiments were approved by the Ethics Committee of Animal Treatment of Fudan University. All reagents were purchased from Sinopharm Chemical Regents (Shanghai, China), unless indicated otherwise. All procedures performed on the rats were in accordance with the European Union protocols (86/609/EEC) for the care and use of laboratory animals.

Selective denervation. The rats were anaesthetized with pentobarbital $(50 \mathrm{mg} / \mathrm{kg})$. The right hind limb was prepared for surgery and a 1-cm incision was made in the skin along the axis of the femur. The nerve branches supplying the soleus and EDL muscles were separated, ligated and truncated distally to the muscles; this step was performed by visualization using a dissecting microscope. The nerve stumps were marked by a knot and buried in the muscles close by to avoid reinnervation, as described previously with modifications $(11,12)$. Sham surgery, which involved the same surgical steps without transection of the nerve, was performed on the contralateral hind limbs of the animals simultaneously, and the soleus and EDL muscles served as the control. The denervation was verified by the location of the knots when the muscles were dissected at $7,14,28$ or 56 days. At the end of the assigned period, the soleus and EDL muscles were carefully excised from the hind limbs, divided into two sections (one for morphological analysis and the other for analyses of protein expression and enzyme activity), cleaned of tendons and connective tissues, and immediately frozen in liquid nitrogen. The muscles were stored at $-80^{\circ} \mathrm{C}$ until processing.

ATPase staining. For the myosin ATPase staining, frozen serial cross sections (thickness, $8 \mu \mathrm{m}$ ) were prepared and staining was performed according to the method described by Brooke and Kaiser (13). ATPase stained sections were observed using an Olympus BX60 microscope (Olympus Corporation, Tokyo, Japan). Those fibers that stained dark following pre-incubation in acid and light following pre-incubation in alkali were classified as type I fibers.

Immunohistochemistry. Freshly obtained skeletal muscle slices (thickness, $8 \mu \mathrm{m}$ ) were fixed at $-20^{\circ} \mathrm{C}$ in $4 \%$ formaldehyde. Prior to antibody staining, the tissue sections were treated with $3 \% \mathrm{H}_{2} \mathrm{O}_{2}$ for 10 min and blocked with $10 \%$ bovine serum for $1 \mathrm{~h}$. The sections were incubated with an in-house generated anti-CAIII antibody $(1: 1,000)(14)$ at $25^{\circ} \mathrm{C}$ for $2 \mathrm{~h}$. Subsequently, the sections were incubated with peroxidase-labeled goat anti-rabbit IgG (1:2,000, ab6721, Abcam, Cambridge, UK) at $25^{\circ} \mathrm{C}$ for $1 \mathrm{~h}$. CAIII was visualized with diaminobenzidene solution $\left(0.5 \mathrm{mg} / \mathrm{ml} ; 0.03 \% \mathrm{H}_{2} \mathrm{O}_{2}\right)$. Stained sections were observed using an Olympus BX60 microscope (Olympus Corporation).

Western blot analysis. The skeletal muscles were homogenized in $10 \mathrm{mM}$ sodium phosphate buffer ( $\mathrm{pH}$ 7.4) containing a mixture of protease inhibitors. The homogenate was centrifuged at $10,000 \mathrm{x} \mathrm{g}$ at $4^{\circ} \mathrm{C}$ for $20 \mathrm{~min}$. The supernatant was collected and the protein concentration was determined using Bradford's method with bovine serum albumin as the standard. For the western blot assays, crude protein extracts $(15 \mu \mathrm{g})$ were separated by $10 \%$ sodium dodecyl sulfate polyacrylamide gel electrophoresis and transferred onto nitrocellulose membranes. The nitrocellulose membranes were blocked with $2.5 \%$ bovine serum albumin at $4^{\circ} \mathrm{C}$ overnight and then incubated with rabbit anti-rat CAIII antibody $(1: 1,600)$ for $3 \mathrm{~h}$ at $25^{\circ} \mathrm{C}$. This was followed by incubation with a peroxidase-conjugated goat anti-rabbit IgG (Chemicon; EMD Millipore, Billerica, MA, USA; $1: 2,000)$ for $2 \mathrm{~h}$ at $25^{\circ} \mathrm{C}$. GAPDH (1:1,200, AG019, Beyotime Institute of Biotechnology, Shanghai, China) served as the internal reference.

Phosphatase staining. Staining for phosphatase activity was performed as reported previously (14). Briefly, following sodium dodecyl sulfate polyacrylamide gel electrophoresis in an ice bath, the proteins were transferred onto a nitrocellulose membrane on ice. The membrane was incubated in ABS buffer containing $20 \mathrm{mM}$ sodium acetate; $0.8 \%$ (w/v) sodium chloride and $0.02 \%(\mathrm{w} / \mathrm{v})$ potassium chloride $\left(\mathrm{pH} \mathrm{5.5)}\right.$ ) at $25^{\circ} \mathrm{C}$ for $20 \mathrm{~min}$ to permit removal of sodium dodecyl sulfate and refolding of the proteins. Subsequently, 5\% (w/v) polyvinylpyrrolidone (40,000 kDa; Sigma-Aldrich; Merck Millipore, Darmstadt, Germany) in ABS buffer was applied at $4^{\circ} \mathrm{C}$ overnight to block the non-specific binding sites. Following washing with ABS buffer for $5 \mathrm{~min}$, the membrane was dipped into a 20-ml staining system ( $\mathrm{pH} 5.5)$ with final concentrations of $50 \mathrm{mM}$ sodium acetate, $20 \mathrm{mM}$ magnesium chloride and 


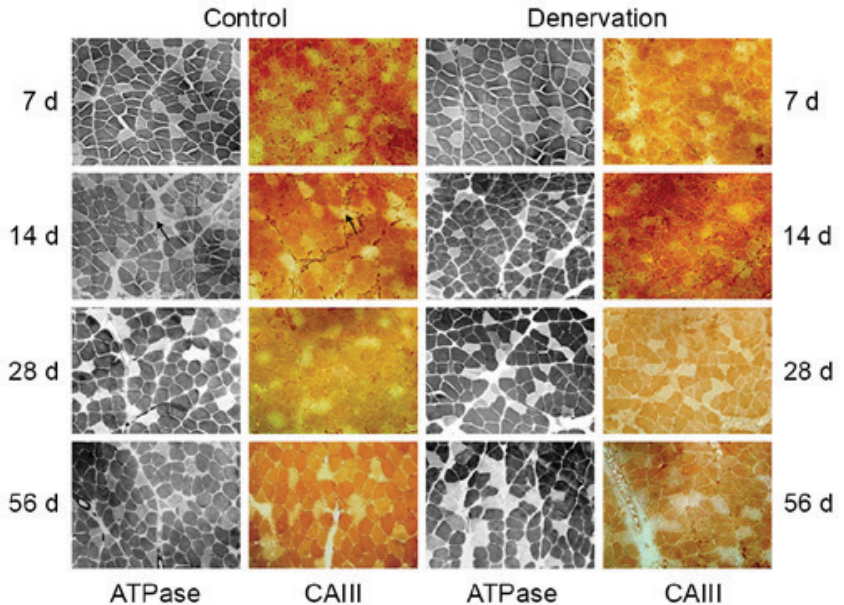

Figure 1. Comparative analysis of staining for ATPase and immunohistochemical staining of CAIII in serial sections of soleus muscles (magnification, $\mathrm{x} 200$ ). Type I myofibers are shown by dark staining for ATPase at $\mathrm{pH} 4.6$. Correlation between ATPase staining and CAIII immunostaining of myofibers in serial sections are shown by solid arrows. No significant changes in fiber morphology or in the ratio of slow- to fast-twitch fibers were observed in the control or denervated soleus muscles. CAIII, carbonic anhydrase III; d, days.

$5 \mathrm{mM}$ nitrophenyl phosphate (Fluka; Merck Millipore), and 2 mM Fast Garnet GBC (Sigma-Aldrich; Merck Millipore). The staining reaction was terminated following incubation at $37^{\circ} \mathrm{C}$ for $45 \mathrm{~min}$.

Statistical analysis. The results of the western blot analysis and phosphatase staining were analyzed and quantified using Image Pro-Plus 6.0 software (Media Cybernetics, Inc., Rockville, MD, USA). The expression of CAIII was normalized to that of GAPDH. Data are expressed as the mean \pm standard error of the mean. Differences between denervated muscles and sham controls were analyzed using Student's paired t-test. $\mathrm{P}<0.05$ was considered to indicate a statistically significant difference.

\section{Results}

Effect of denervation on skeletal muscle structure and expression of CAIII. Comparative analyses of the staining for ATPase and immunohistochemical staining of CAIII were performed on serial sections of the soleus and EDL muscles. The soleus muscles were composed mainly of type I fibers, as shown by the dark ATPase staining at $\mathrm{pH} 4.6$ (Fig. 1). Throughout the 56-day observation period, there was a decline in type I fibers, but no significant change in fiber morphology or the ratio of slow-twitch fibers in the control or denervated soleus muscle $(\sim 83 \%$ of type I fibers in the control muscles and $\sim 80 \%$ of type I fibers in the denervated muscles at day 56; $\mathrm{P}>0.05)$. In addition, there was no visible atrophy, hypertrophy or hyperplasia of connective tissues. Immunohistochemical staining of the soleus muscle for CAIII showed that the location of the CAIII enzyme was consistent with the type I fibers in the control group, and this was maintained following denervation (Fig. 1).

The EDL muscle was composed mainly of fast-twitch fibers and a small number of type I fibers (Fig. 2). Throughout the observation period, no significant change was observed in

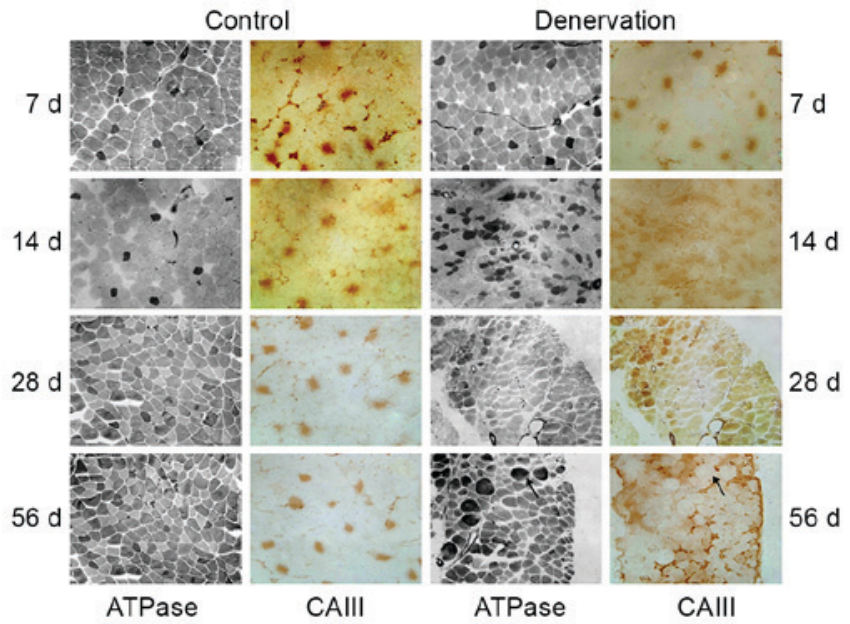

Figure 2. Comparative analysis of staining for ATPase and immunohistochemical staining of CAIII in serial sections of EDL muscles (magnification, $\mathrm{x} 200$ ). Following denervation, the morphology of EDL muscles changed significantly. In the first 2 weeks, the number of type I fibers increased gradually. Subsequent reconstruction was demonstrated by non-selective ATPase staining ( $28 \mathrm{~d}$ and $56 \mathrm{~d}$ post denervation). On day 56 post-denervation, discrepancy between ATPase staining and CAIII immunohistochemical staining (indicated by solid arrows) was observed. CAIII, carbonic anhydrase III; EDL, extensol digitorum longus; d, days.

the ratio of the two myofiber types, the consistency of type I myofibers or the location of CAIII in the control EDL tissues. Following denervation, the morphology of the EDL muscle changed significantly. Firstly, all denervated muscle fibers (both type I and other fibers) gradually lost their polygonal appearance and became rounded, changing from uniform shapes to different sizes. Secondly, the composition ratio of the muscle fibers changed following denervation. In the first 2 weeks, the number of type I fibers gradually increased, indicating the conversion from fast- to slow-twitch fibers. Further reconstruction was demonstrated by non-selective ATPase staining, which indicated the appearance of transitional type fibers. During this time course, the expression of CAIII changed accordingly. In the first 2 weeks, the number of CAIII-immunoreactive fibers increased, and the location of the fibers was consistent with the fast-to-slow skeletal muscle remodeling. During the subsequent 2 weeks, in addition to the dedifferentiation of EDL muscle, with the exception of typical type I fibers, CAIII immunostaining became more diffuse over time. At 56 days post-denervation, a mismatch between ATPase staining and CAIII immunohistochemical staining was observed.

Effect of denervation on the protein expression of CAIII in skeletal muscles. In the control group, the protein levels of CAIII in the soleus and EDL muscles increased gradually over time. This trend was more marked in the soleus than in the EDL muscle, and the level of CAIII in the soleus was markedly higher than that in the EDL muscle. Following denervation, the levels of CAIII in the soleus and EDL muscles were higher, compared with the levels in the control groups (Fig. 3). Compared with the increased protein level of CAIII in the EDL muscle over time following denervation, the level in the soleus muscle was significantly increased at 2 weeks but then decreased gradually, reaching control levels 
Table I. Effect of denervation on the phosphatase activity of carbonic anhydrase III in skeletal muscles.

\begin{tabular}{lccccc}
\hline Group & $\mathrm{n}$ & Day 7 & Day 14 & Day 28 & Day 56 \\
\hline Soleuscontrol & 6 & $16.75 \pm 1.25$ & $22.75 \pm 1.80$ & $25.26 \pm 3.15$ & $25.82 \pm 2.97$ \\
Soleusdenervation & 6 & $13.29 \pm 0.94$ & $14.39 \pm 1.93^{\mathrm{a}}$ & $11.48 \pm 1.46^{\mathrm{a}}$ & $9.04 \pm 1.46^{\mathrm{a}}$ \\
EDLcontrol & 6 & $0.98 \pm 0.19$ & $1.06 \pm 0.21$ & $1.15 \pm 0.16$ & $1.12 \pm 0.16$ \\
EDLdenervation & 6 & $0.67 \pm 0.09$ & $0.96 \pm 0.21$ & $0.98 \pm 0.19$ & $0.52 \pm 0.09^{\mathrm{b}}$ \\
\hline
\end{tabular}

${ }^{\mathrm{a}} \mathrm{P}<0.05$, vs. soleus control; ${ }^{\mathrm{b}} \mathrm{P}<0.05$, vs. EDL control. EDL, extensol digitorum longus.

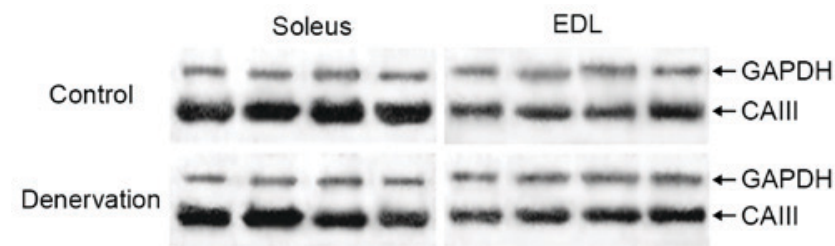

$7 d 4$ d 28 d 56 d 7 d 14 d 28 d $56 d$

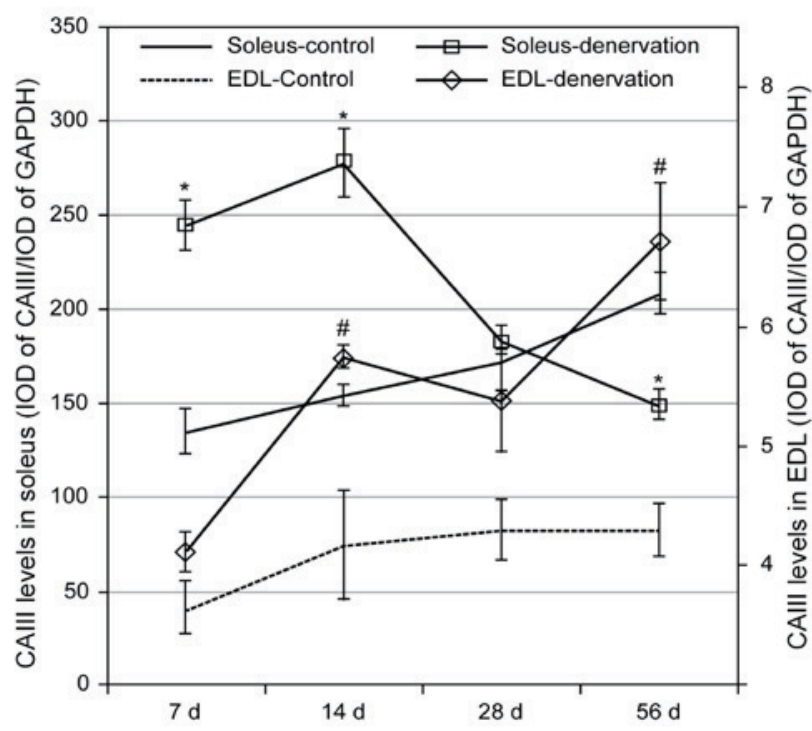

Figure 3. Effect of denervation on protein expression of CAIII in skeletal muscles. Differences between the denervated muscles and the sham controls were analyzed using a paired Student's t-test. ${ }^{*} \mathrm{P}<0.05$ vs. Soleus control, ${ }^{\#} \mathrm{P}<0.05$ vs. EDL control. CAIII, carbonic anhydrase III; IOD, integral optical density; EDL, extensol digitorum longus.

at 28 days and dropping below the control level at 56 days $(\mathrm{P}<0.05)$

Effect of denervation on the phosphatase activity of CAIII in skeletal muscle. The phosphatase activity of CAIII in the control group increased with time in the soleus and EDL muscles, maintaining a steady enzyme activity-protein ratio (Table I). Following denervation, the phosphatase activity of CAIII in the soleus and EDL muscles decreased sharply, compared with the previously observed increase in CAIII. This decrease was more marked with time, particularly in the soleus muscle $(\mathrm{P}<0.05)$. These data indicated a deviation between the phosphatase activity and protein expression of CAIII in denervated muscle (Fig. 4A and B).
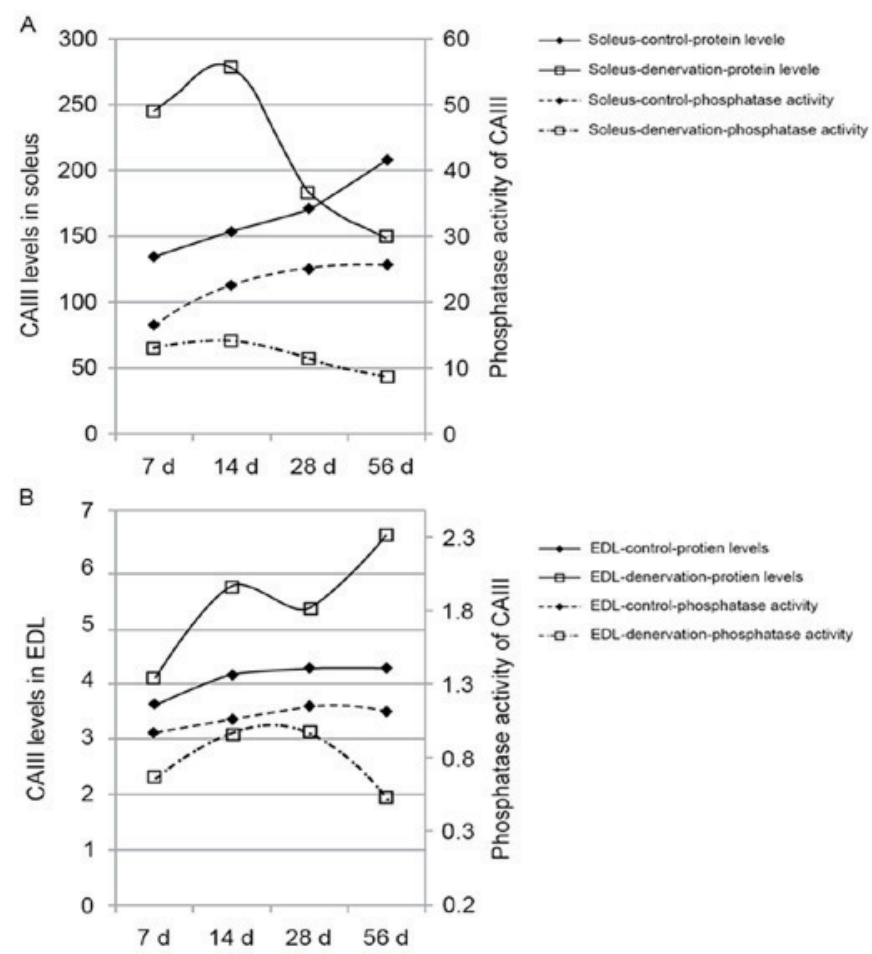

Figure 4. Comparative analysis of changes in the expression and phosphatase activity of CAIII following denervation. (A) Soleus muscle (B) EDL. CAIII, carbonic anhydrase III; EDL, extensol digitorum longus; d, days.

\section{Discussion}

The present study adopted a selective denervation technique to assess the impact of nerve conduction on CAIII. Comparative analyses to examine relative changes in muscle structure and CAIII location, and to evaluate the expression of CAIII and its phosphatase activity following denervation, revealed that: i) CAIII was distributed selectively in type I myofibers; ii) following denervation, skeletal muscle fibers of the EDL muscle exhibited significant type transformation (remodeling). This was followed by a change in the localization of CAIII, which was more pronounced in the EDL muscle than in the soleus muscle. Specifically, there was a fast-to-slow conversion of myofibers staining positively for active CAIII; iii) Protein levels of CAIII increased to varying extents in denervated muscles, whereas its phosphatase activity decreased significantly. Innervation is critical for myofiber differentiation, maturation and maintenance. Denervation in animals is a common approach 
to assess the impact of innervation on skeletal muscle structure and functions. Traditionally, sciatic denervation causes near paralysis of the entire limb and leads to fiber atrophy, including denervation and disuse atrophy. Myofiber remodeling from fast-to-slow or slow-to-fast type is also observed as a result of the conversion of original myofibers and/or newly-formed fibers from satellite cells $(15,16)$. This change is more markedin the soleus than in the EDL muscle (17).

In certain studies, selective denervation of a specific muscle has been performed, which differs from traditional surgery $(12,18)$. In the present study, following selective denervation, no significant structural change was observed in the soleus muscle. By contrast, prominent muscle remodeling occurred shortly following denervation of the EDL muscle, demonstrating that fast fibers were more susceptible to atrophy than slow fibers (19). Pathologically, the denervated EDL fibers lost their polygonal shape and, over time, the fibers began to exhibit dedifferentiation (17). These different effects of selective denervation maybe associated with the absence of disruption from mechanical forces and blood supply, which exist in traditional animal denervation models $(10,20,21)$. It was also apparent that the necessity of innervation for maintaining muscle morphology is more important in the EDL muscle than in the soleus muscle. Considering the differences between the different denervation animal models, even within the same study $(12,21)$, it is reasonable and important to consider and evaluate the differing effects between selective and non-selective denervation in a selected animal model.

As reported previously, CAIII is expressed selectively in type I myofibers (5). Following denervation, the localization of CAIII changed in response to myofiber remodeling, and this effect was significantly different between the soleus and EDL muscles. No significant change was observed in the structure of the soleus muscle following denervation. This was consistent with the absence of changes in the distribution of type I myofibers and the localization of CAIII during the observation period. However, the tissue structure and immunostaining for CAIII altered significantly in the denervated EDL muscle. In the first 2 weeks (early stage), the localization of CAIII-positive fibers was consistent with the localization of type I fibers. At later time points, the ATPase staining showed no complete or clear distinguished skeletal muscle fiber types, indicating dedifferentiation $(17,18)$. In addition, there was a lack of complete overlap between CAIII immunostaining and ATPase staining, indicating the existence of transitional myofibers (22) in which the cells may or may not express CAIII. Although immunostaining for CAIII and myosin heavy chain isoforms was not applied to the serial sections to describe the relative changes of these two indices, the present study did show consistent changes in the location of CAIII and type I myofiber (slow-twitch fiber) distribution.

The remodeling of skeletal muscle is an adaptive change in response to physiological and pathological signals (23). Fast-slow and slow-fast transformations occur through intracellular signal transduction, resulting in a change in the gene expression of specific myosin heavy chain isoforms $(15,24)$. In the present study, CAIII was localized in type I myofibers, and the change of its location was consistent with the change in type I myofibers following denervation. Therefore, it is possible that
CAIII is regulated by the signaling pathway, which regulates slow fiber programming (25).

The transformation of muscle structure (CAIII and myofibers) was confirmed in the present study by analyzing the protein levels of CAIII. Following denervation, the protein levels of CAIII increased in the slow- and fast-twitch fibers at an early stage, but long-term changes appeared to differ. Specifically, at day 14, the protein levels of CAIII began to decline in the denervated soleus muscle, whereas the levels in the denervated EDL muscle continued to increase gradually. This difference may have been caused by the increased release of CAIII from reserves (i.e., the transcriptional pool) in the early stage following denervation, followed by the de novo synthesis of CAIII through gene transcription and translation. The levels of CAIII are determined by the number of type I myofibers (CAIII-immunostained myofibers). The number of type I myofibers in the EDL muscle increased gradually, leading to a concomitant increase in the level of CAIII.

In contrast to the differential changes in protein levels, the phosphatase activity of CAIII decreased significantly in the two types of denervated muscles. Changes in neurotrophic and contraction activity occur in denervated muscles, resulting in decreases in muscle metabolism associated with changes in the levels of enzymes and contraction-associated proteins $(26,27)$. The selective expression of CAIII in skeletal muscles, liver tissues and adipose tissues suggests that it is directly or indirectly involved in energy metabolism (5). Therefore, the significant reduction inCAII phosphatase activity following denervation may be an adaptive response to reduced skeletal muscle contraction and cell metabolism. Intracellular redox reactions and dephosphorylation/phosphorylation in skeletal muscles are also affected by denervation (28), and the change in CAIII phosphatase activity maybe a reflection of these changes (29).

In conclusion, the present study demonstrated that the expression and activity of CAIII, a contraction-associated enzyme, were regulated by innervation. Following denervation, the expression and activity of CAIII were altered with myofiber remodeling. The differences in manifestations observed in the present study from those in previous studies on muscle structure following denervation maybe the result of the maintenance of passive movements of the denervated muscles in the present study. Therefore, further investigations are required to assess the effects of movement inhibition, including tenotomy or suspension, on CAIII. Although the mechanism requires further investigation, the changes in the expression of CAIII observed in the present study reflect changes in type I myofibers, indicating that CAIII is important for muscle contraction, particularly in resistance to fatigue.

\section{Acknowledgements}

The present study was supported by the Natural Science Foundation of China (grant no. 30170329).

\section{References}

1. Ayoglu B, Chaouch A, Lochmüller H, Politano L, Bertini E, Spitali P, Hiller M, Niks EH, Gualandi F, Pontén F, et al: Affinity proteomics within rare diseases: A BIO-NMD study for blood biomarkers of muscular dystrophies. EMBO Mol Med 6: 918-936, 2014. 
2. Olausson P, Gerdle B, Ghafouri N, Sjöström D, Blixt E and Ghafouri B: Protein alterations in women with chronic widespread pain-An explorative proteomic study of the trapezius muscle. Sci Rep 5: 11894, 2015.

3. Chen CN, Ferrington DA and Thompson LV: Carbonic anhydrase III and four-and-a-half LIM protein 1 are preferentially oxidized with muscle unloading. J Appl Physiol (1985) 105: 1554-1561, 2008.

4. Du AL, Ren HM, Lu CZ, Tu JL, Xu CF and Sun YA: Carbonic anhydrase III is insufficient in muscles of myasthenia gravis patients. Autoimmunity 42: 209-215, 2009.

5. Harju AK, Bootorabi F, Kuuslahti M, Supuran CT and Parkkila S: Carbonic anhydrase III: A neglected isozyme is stepping into the limelight. J Enzyme Inhib Med Chem 28: 231-239, 2013.

6. Côté $\mathrm{CH}$, Ambrosio F and Perreault G: Metabolic and contractile influence of carbonic anhydrase III in skeletal muscle is age dependent. Am J Physiol 276: R559-R565, 1999.

7. Cabiscol E and Levine RL: Carbonic anhydrase III. Oxidative modification in vivo and loss of phosphatase activity during aging. J Biol Chem 270: 14742-14747, 1995.

8. Shang X, Chen S, Ren H, Li Y and Huang H: Carbonic anhydrase III: The new hope for the elimination of exercise-induced muscle fatigue. Med Hypotheses 72: 427-429, 2009.

9. Milot J, Frémont P, Côté C and Tremblay RR: Differential modulation of carbonic anhydrase (CA III) in slow- and fast-twitch skeletal muscles of rat following denervation and reinnervation. Biochem Cell Biol 69: 702-710, 1991.

10. Argadine HM, Hellyer NJ, Mantilla CB, Zhan WZ and Sieck GC: The effect of denervation on protein synthesis and degradation in adult rat diaphragm muscle. J Appl Physiol (1985) 107: 438-444, 2009.

11. Lu DX, Huang SK and Carlson BM: Electron microscopic study of long-term denervated rat skeletal muscle. Anat Rec 248 355-365, 1997.

12. Ashley Z, Sutherland H, Lanmüller H, Russold MF, Unger E, Bijak M, Mayr W, Boncompagni S, Protasi F, Salmons S and Jarvis JC: Atrophy, but not necrosis, in rabbit skeletal muscle denervated for periods up to one year. Am J Physiol Cell Physiol 292: C440-C451, 2007.

13. Brooke MH and Kaiser KK: Muscle fiber types: How many and what kind? Arch Neurol 23: 369-379, 1970.

14. Huang H, Ren HM, Shang XL and Liu XY: Detection of the phosphatase activity of carbonic anhydrase III on a nitrocellulose membrane following 2D gel electrophoresis. Mol Med Rep 10: 1887-1892, 2014

15. Bassel-Duby R and Olson EN: Signaling pathways in skeletal muscle remodeling. Annu Rev Biochem 75: 19-37, 2006.
16. Dumont NA, Bentzinger CF, Sincennes MC and Rudnicki MA Satellite cells and skeletal muscle regeneration. Compr Physiol 5: $1027-1059,2015$

17. Midrio M: The denervated muscle: Facts and hypotheses. A historical review. Eur J Appl Physiol 98: 1-21, 2006.

18. Szabó A, Wuytack F and Zádor E: The effect of passive movement on denervated soleus highlights a differential nerve control on SERCA and MyHC isoforms. J Histochem Cytochem 56: 1013-1022, 2008.

19. Viguie CA, Lu DX, Huang SK, Rengen H and Carlson BM: Quantitative study of the effects of long-term denervation on the extensor digitorum longus muscle of the rat. Anat Rec 248: 346-354, 1997.

20. Eisenberg HA and Hood DA: Blood flow, mitochondria, and performance in skeletal muscle after denervation and reinnervation. J Appl Physiol (1985) 76: 859-866, 1994.

21. Adhihetty PJ, O'Leary MF, Chabi B, Wicks KL and Hood DA: Effect of denervation on mitochondrially mediated apoptosis in skeletal muscle. J Appl Physiol (1985) 102: 1143-1151, 2007.

22. Patterson MF, Stephenson GM and Stephenson DG: Denervation produces different single fiber phenotypes in fast- and slow-twitch hindlimb muscles of the rat. Am J Physiol Cell Physiol 291: C518-C528, 2006

23. Blaauw B, Schiaffino S and Reggiani C: Mechanisms modulating skeletal muscle phenotype. Compr Physiol 3: 1645-1687, 2013.

24. Cohen TJ, Choi MC, Kapur M, Lira VA, Yan Z and Yao TP: HDAC4 regulates muscle fiber type-specific gene expression programs. Mol Cells 38: 343-348, 2015.

25. Mitterberger MC, Kim G, Rostek U, Levine RL and Zwerschke W: Carbonic anhydrase III regulates peroxisome proliferator-activated receptor- $\gamma 2$. Exp Cell Res 318: 877-886, 2012.

26. Tang H, Cheung WM, Ip FC and Ip NY: Identification and characterization of differentially expressed genes in denervated muscle. Mol Cell Neurosci 16: 127-140, 2000.

27. Sakuma K, Watanabe K, Sano M, Kitajima S, Sakamoto K, Uramoto I and Totsuka T: The adaptive response of transforming growth factor-beta 2 and -beta RII in the overloaded, regenerating and denervated muscles of rats. Acta Neuropathol 99: $177-185,2000$

28. Abruzzo PM, di Tullio S, Marchionni C, Belia S, Fanó G, Zampieri S, Carraro U, Kern H, Sgarbi G, Lenaz G and Marini M: Oxidative stress in the denervated muscle. Free Radic Res 44: 563-576, 2010.

29. Räisänen SR, Lehenkari P, Tasanen M, Rahkila P, Härkönen PL and Väänänen HK: Carbonic anhydrase III protects cells from hydrogen peroxide-induced apoptosis. FASEB J 13: 513-522, 1999. 